\title{
FMH und FMH Services an der IFAS
}

\author{
E. Flückiger
}

Vom 22.-25. Oktober 2002 findet an der Messe Zürich die 27. Internationale Fachmesse für Ärzte und Spitalbedarf statt. FMH und FMH Services laden Sie ein zu einer Reihe aktueller Informationsveranstaltungen. Diese Workshops können einzeln oder als Ganzes besucht werden.

Das ist die Auswahl:

\section{Steueroptimierung mit Versicherungsprodukten}

Dienstag, 22., und Donnerstag, 24. Oktober 2002, jeweils 10.00-10.45 Uhr

Referent: Peter Gygax, FMH Insurance \& Financial Services

Aus den Inhalten: «Einkäufe in die Pensionskasse», «Gebundene Vorsorge 3a»,

«Konservative Kapitalanlagen», «Todesfallrisiko-Policen» u.a.m.

\section{Vorzeitige Pensionierung/Finanzplanung}

Dienstag, 22., und Donnerstag, 24. Oktober 2002, jeweils 11.15-12.00 Uhr

Referent: Peter Gygax, FMH Insurance \& Financial Services

Aus den Inhalten: «Sicherung des Lebensstandards im Alter»,

«Reichen meine Ersparnisse bis ans Lebensende», «Zeitgerechter Aufbau der persönlichen Altersvorsorge», «Frühpensionierung», «PK-Guthaben: Als Kapital oder Rente planen?», «Steueroptimierungen» u.a.m.

\section{Die Arztpraxis aus steuerlicher Sicht}

Dienstag, 22., und Donnerstag, 24. Oktober 2002, jeweils 14.00-14.45 Uhr

Referent: Urs Gross, FMH Services Treuhand

Aus den Inhalten: «Steuereinsparungen durch Gewinn-Nivellierung» und «MWST-Pflicht bei Ärztinnen und Ärzten»

\section{Ausstehende Honorarforderungen. Was nun?}

Dienstag, 22., und Donnerstag, 24. Oktober 2002, jeweils 15.15-16.00 Uhr

Referentin: Margrith Fankhauser, FMH Inkasso Services

Aus den Inhalten: «Prävention», «Mahnen mit System», «Zeitpunkt für Inkassoauftrag», «Schuldbetreibung auf Pfändung und Konkurs», «Vorgehen bei Rechtsvorschlag und Pfändungsverfahren», «Wirkung/Bedeutung des Datenschutzes (Arztgeheimnis und Inkassoverfahren)»

\section{Gesicherte E-Mail-Kommunikation zwischen Spital und Praxis}

Mittwoch, 23., und Freitag, 25. Oktober 2002, jeweils 10.00-10.45 Uhr

Referent: Maurizio Campagnaro, Health-Info-Net (HIN)

Aus den Inhalten: «Ansprüche aus dem Datenschutzgesetz an die Kommunikation sensibler und vertraulicher Daten», «Allgemeine Gefahren der E-Mail-Kommunikation», «HIN-Lösungen», «Praktische (reelle) Beispiele aus der HIN-Technologie» u.a.m. 


\section{«Werbung» und Websites für Arztpraxen}

Mittwoch, 23., und Freitag, 25. Oktober 2002, jeweils 11.15-12.00 Uhr

Referent/in: Lahor Jakrlin und Ursula Rytz, FMH Marketing \& Kommunikation SA

Aus den Inhalten: «Beispiele von Kommunikationslösungen» sowie «Möglichkeiten und Rahmenbedingungen bei der Erstellung von professionellen Internetlösungen».

\section{Praxisübernahme: Worauf Sie achten müssen}

Mittwoch, 23., und Freitag, 25. Oktober 2002, jeweils 14.00-14.45 Uhr

Referent: Robert Gmür, lic. iur., FMH Rechtsdienst

Aus den Inhalten: «Zulassungsstopp auch für Übernahmen?» «Was ist mit den Krankengeschichten?» «Wie einige ich mich mit Vermieter und Personal?»

\section{Die ärztliche Gruppenpraxis: Tips zur Vertragsgestaltung}

Mittwoch, 23., und Freitag, 25. Oktober 2002, jeweils 15.15-16.00 Uhr

Referent: Robert Gmür, lic. iur., FMH Rechtsdienst

Aus den Inhalten: «Poollösung oder Apparategemeinschaft?» «Einsteigen als Junior-Partner/in? AG oder einfache Gesellschaft?»

\section{Anmeldung und Teilnahme}

Kosten

Für FMH-Mitglieder ist die Teilnahme kostenlos. Nichtmitglieder der FMH entrichten einen Unkostenbetrag von Fr. 30.- pro Workshop (Barbezahlung bei der Eingangskontrolle zu den Workshops). Inbegriffen: Alle Teilnehmerinnen und Teilnehmer erhalten einen GRATIS-Eintritt an die IFAS (Wert Fr. 20.-).

\section{Anmeldungen}

Anmeldungen sind mit Name/Vorname, genauer Adresse, FMH-Mitgliedernummer, Workshop-Titel und gewünschtem Datum bis spätestens 18. Oktober 2002 an Erika Flückiger, Leiterin Dienste FMH, Elfenstrasse 18, Postfach 293, 3000 Bern 16, zu richten. E-Mail: efluecki@hin.ch

Die Registrierung erfolgt in der Reihenfolge der eingegangenen Anmeldungen. 